\title{
Two-dimensional Kalman filter approach to airborne vector gravimetry
}

DOI: https://doi.org/10.1515/jogs-2019-0009

Received November 29, 2018; accepted February 4, 2019

\begin{abstract}
The paper presents a new approach to the airborne vector gravimetry problem. The idea of the approach is to take into account spatial correlation of the gravity field to improve observability of horizontal components of the gravity disturbance vector (GDV). We consider the GDV determination problem given airborne data at a set of parallel survey lines assuming that lines are flown in the same direction at a constant height above the reference ellipsoid. We use a 2-D random field model for the gravity field at the flight height. The random field is governed by two autoregressive equations (one in the direction along the lines, the other across the lines). Then we pose the estimation problem simultaneously for the GDV horizontal components and systematic errors of an inertial navigation system at all the lines simultaneously. The developed estimation algorithm is based on 2D Kalman filtering and smoothing techniques. Numerical results obtained from simulated data processing showed improved accuracy of the gravity horizontal component determination.
\end{abstract}

Keywords: airborne vector gravimetry, gravity disturbance vector, random fields, Kalman filter

\section{Introduction}

Airborne gravimetry is a method of measuring the Earth's gravity field. The gravity information is of high importance for geodesy, geophysics, navigation, geodynamics, and others. While other gravity measurement methods have limitations either in spatial resolution of recovered gravity or due to the type of terrain, airborne gravimetry technique is capable to provide gravity data of high accuracy and resolution at any area of interest. Currently, gravity data provided by airborne gravimetry have spatial resolution of $2-$

\footnotetext{
*Corresponding Author: V.S. Vyazmin: Laboratory of Navigation and Control, Faculty of Mechanics and Mathematics, Lomonosov Moscow State University, Moscow, Russia

Yu.V. Bolotin: Department of Applied Mechanics and Control, Faculty of Mechanics and Mathematics, Lomonosov Moscow State University, Moscow, Russia
}

$5 \mathrm{~km}$ and accuracy of $0.5-1 \mathrm{mGal}$ (Kennedy et al. 2002; Olson 2010).

Airborne gravimetry is well-established for measuring the vertical component of the gravity disturbance vector (GDV), i.e. gravity disturbance (scalar gravimetry) (Childers et al. 1999; Bolotin and Golovan 2013). In the last two decades, the airborne vector gravimetry, which measures the total GDV, is actively developed by a number of authors (Kwon and Jekeli 2001; Schwarz et al. 2002; Cai et al. 2013; Becker et al. 2016). Unlike scalar gravimetry, this method requires significantly more accurate inertial navigation system (INS). Horizontal orientation errors of 30 arc seconds, which are acceptable in airborne scalar gravimetry, will yield an error of $150 \mathrm{mGal}$ in the GDV horizontal components (or, equivalently, 30 arc seconds in the deflections of the vertical).

In addition to the instrument accuracy, there are specific requirements to processing algorithms. The main difficulty of the airborne vector gravimetry problem lies in low observability of the gravity horizontal components. These are observable only in combination with the INS systematic errors such as the accelerometer biases and the orientation errors produced by the gyroscope errors. To separate the gravity horizontal components from the INS systematic errors, an additional information on gravity or the INS systematic errors (i.e. regularization) is required.

The traditional way of introducing additional information on gravity in the airborne vector gravimetry is based on using stochastic processes in the time domain to represent each GDV component (Jekeli 1994; Becker et al. 2016). Different gravity stochastic models were proposed such as Gauss-Markov processes or more complex models that, e.g. take into account consistency of the GDV components (Jordan 1972; Forsberg 1987). However, this approach does not provide full separation of the GDV horizontal component estimates and the INS systematic errors. A number of alternative approaches were proposed.

An interesting approach free from any gravity model was presented in Kwon and Jekeli (2001). It is based on processing of several repeated lines flown over the same ground track assuming that the INS systematic errors related to different lines are uncorrelated and that gravity 
signal is common at these lines. Decorrelation of the gravity measurements is performed in the frequency domain, and the residual INS errors are filtered out of the GDV estimates. The approach showed much better accuracy compared with the traditional one (Kwon and Jekeli 2001).

An approach presented in Cai et al. (2013) is based on using data of the global gravity field model EGM2008 (Pavlis et al. 2012) to separate the residual INS systematic errors and along-line estimates of the GDV horizontal components. A simple linear-trend model was used for the INS errors. Numerical results of this method are promising (Cai et al. 2013).

As an additional information, extra observations can be used to reduce the orientation errors [e.g. star trackers (Dai et al. 2014; Mangold 1997)].

In this paper, we propose a new approach to the GDV determination based on 2- D stochastic modeling of the gravity field in the survey area. Main idea of our approach is to take into account spatial correlations of the gravity field to improve observability of the gravity horizontal components. We consider the INS error propagation equations and pose the GDV determination problem given airborne measurements along a set of parallel lines at constant height above the reference ellipsoid. It is assumed that the lines are flown in the same direction at constant aircraft speed and that airborne measurements along any two lines are time-synchronized.

We introduce a homogeneous 2-D random field to represent the gravity field at the flight height. The random field is governed by two autoregressive equations of order two, one of which is directed along the lines, the other across the lines. In the two-dimensional frequency domain, the random field is characterized by a 2-D power spectral density (PSD) that can be represented as a product of two one-dimensional ones. Coefficients of the autoregressive equations can be determined given an a priori value of the gravity correlation radius.

Under the assumptions made, we reduce the considered problem to optimal estimation of only the gravity disturbance horizontal components. Several adjacent survey lines are selected for simultaneous processing. The problem is solved via Kalman filtering and smoothing. The state vector for the filter includes variables of the INS error propagation equations and the disturbing potential values at the same time instant of each adjacent line. The estimation algorithm includes the following steps:

1) estimation of the GDV horizontal components and the INS systematic errors along adjacent lines via Kalman filtering;

2) smoothing of the obtained estimates;
3) repetition of the steps 1-2 for the following set of adjacent lines.

The number of adjacent lines can be chosen from 3 to the total number of the survey lines. In the last case, the estimation algorithm is optimal under the minimum mean squared error criterion in the class of linear algorithms. Note that the algorithm does not require repeated survey lines.

An accuracy analysis of the developed approach was presented in Bolotin and Vyazmin (2018). In this paper, a detailed theoretical development of the estimation algorithm is presented. Numerical results obtained from semisimulated data processing are discussed.

\section{Airborne vector gravimetry equations}

Let $\mathrm{M}$ be the center of the accelerometer triad of a strapdown airborne gravimeter. We will refer to the point $M$ as the gravimeter proof mass. Denote by $M z$ the body (aircraft) coordinate frame with its center in $M$ and its axes coinciding with the sensitivity axes of the accelerometers. As navigation frame, we use the geodetic local level frame $M x$. The axis $M x_{1}$ points east, $M x_{2}$ points north, and $M x_{3}$ points in the direction of the normal to the reference ellipsoid.

We assume throughout this paper that geodetic coordinates (latitude, longitude, altitude) of $M$ are known from post-processing differential phase measurements of global navigation satellite system (GNSS) receivers. We neglect the positioning solution errors, which are assumed to be at the level of several $\mathrm{cm}$ at $20 \mathrm{~Hz}$ (Lu et al. 2017). Such accuracy is sufficient to calculate the normal gravity, centrifugal and Coriolis terms in the INS mechanization equations (Eq. (3) below) up to the accuracy of $0.1 \mathrm{mGal}$.

\section{A. Basic equations of airborne vector gravimetry}

The basic model of airborne gravimetry is the equations of motion of the proof mass $M$ derived from Newton's second law of motion. The equations of motion expressed in the navigation frame $M x$ and the kinematic equation for the body-frame orientation matrix $L_{z x}$ are written as Farrell (2008):

$$
\begin{aligned}
\dot{\boldsymbol{v}}_{x} & =\left(\hat{\boldsymbol{\omega}}_{x \xi}^{x}+\hat{\boldsymbol{\omega}}_{\eta \xi}^{x}\right) \boldsymbol{v}_{x}+L_{x z} \boldsymbol{f}_{z}+\gamma_{x}+\delta \mathbf{g}_{x}, \\
\dot{L}_{z x} & =\hat{\boldsymbol{\omega}}_{z \xi}^{z} L_{z x}-L_{z x} \hat{\boldsymbol{\omega}}_{x \xi}^{x} .
\end{aligned}
$$


Here $\boldsymbol{v}_{x}$ is the aircraft velocity relative to the Earth expressed in the $M x$ frame, $\delta \mathbf{g}_{x}=\left(\delta \mathrm{g}_{x_{1}}, \delta \mathrm{g}_{x_{2}}, \delta \mathrm{g}_{x_{3}}\right)^{T}$ is the $\mathrm{GDV}, \delta \mathrm{g}_{x_{1}}, \delta \mathrm{g}_{x_{2}}, \delta \mathrm{g}_{x_{3}}$ are the east, north and up components, respectively, $\gamma_{x}$ is the normal gravity vector, $\boldsymbol{f}_{z}$ is the specific force in the body frame $M z, L_{x z}=L_{z x}^{T}$ is the transformation matrix from $M z$ to $M x, \boldsymbol{\omega}_{x \xi}^{x}$ is the (absolute) angular velocity of the $x$-frame with respect to the Earth-centered inertial $\xi$-frame, expressed in $M x$ frame coordinates, $\boldsymbol{\omega}_{z \xi}^{z}$ is the absolute angular velocity of $M z$, expressed in $M z$ frame coordinates, $\boldsymbol{\omega}_{\eta \xi}^{X}$ is the Earth angular velocity vector (the absolute angular velocity of the Earthcentered Earth-fixed $\eta$-frame), expressed in $M x$ frame coordinates, $\hat{\boldsymbol{\omega}}_{\eta \xi}^{x}$ is a skew-symmetric matrix formed by the components of the vector $\boldsymbol{\omega}_{\eta \xi}^{x}$ in such a way that $\hat{\boldsymbol{\omega}}_{\eta \xi}^{x} \boldsymbol{v}_{x}=$ $-\boldsymbol{\omega}_{\eta \xi}^{x} \times \boldsymbol{v}_{x}$ where the symbol $\times$ means the cross product.

The INS mechanization equations are obtained from Eq. (1) by replacing true values of the body-frame angular velocity $\boldsymbol{\omega}_{z \xi}^{z}$ and specific force $\boldsymbol{f}_{z}$ with measurements of gyroscopes and accelerometers

$$
\boldsymbol{\omega}_{z \xi}^{z^{\prime}}=\boldsymbol{\omega}_{z \xi}^{z}+\Delta \boldsymbol{\omega}_{z \xi}^{z}, \boldsymbol{f}_{z}^{\prime}=\boldsymbol{f}_{z}+\Delta \boldsymbol{f}_{z} .
$$

We denoted by $\Delta$ the measurement errors. Hence, we have

$$
\begin{aligned}
\dot{\boldsymbol{v}}_{x}^{\prime} & =\left(\hat{\boldsymbol{\omega}}_{x \xi}^{x}+\hat{\boldsymbol{\omega}}_{\eta \xi}^{x}\right) \boldsymbol{v}_{x}^{\prime}+L_{x z}^{\prime} \boldsymbol{f}_{z}^{\prime}+\gamma_{x}, \\
\dot{L}_{z x}^{\prime} & =\hat{\boldsymbol{\omega}}_{z \xi}^{z^{\prime}} L_{z x}^{\prime}-L_{z x}^{\prime} \hat{\boldsymbol{\omega}}_{x \xi}^{x}
\end{aligned}
$$

given initial values for $\boldsymbol{v}_{x}^{\prime}, L_{z x}^{\prime}$. The matrix $L_{z x}^{\prime}$ is the transformation matrix from $M x$ to the computed body frame denoted by $M z^{\prime}$.

Note that there are no equations for the positioning solution in Eq. (3) as we assume that proof mass positions are perfectly known from the GNSS positioning solution. Values of $\boldsymbol{\omega}_{x \xi}^{x}, \boldsymbol{\omega}_{\eta \xi}^{x}, \gamma_{x}$ are calculated from the GNSS positioning and velocity solutions via known formulae neglecting inaccuracies due to GNSS errors (Bolotin and Golovan 2013; Farrell 2008).

Note that the gyroscope error $\Delta \boldsymbol{\omega}_{z \xi}^{z}$ in Eq. (2) can also be defined with negative sign [as, e.g., in Bolotin and Golovan (2013); Bolotin Yu. and Vyazmin (2018)]. The minus sign is selected to make the attitude error equations for platform and strapdown systems identical in form (Huddle 1983).

\section{B. INS error model and observation model}

Let orientation errors of the computed body frame $M z^{\prime}$ be small and representable by a vector of infinitesimal rotation $\boldsymbol{\alpha}_{z}$ (a navigation-grade INS is assumed) (Farrell 2008). Denote by $\boldsymbol{\alpha}_{x}$ the result of a projection $\boldsymbol{\alpha}_{x}=L_{z x}^{\prime} \boldsymbol{\alpha}_{z}$, i. e. an orientation error of a frame, which is close to the geodetic local level frame. Denote by $\delta \boldsymbol{v}_{x}$ the velocity error defined as the sum of the error in the computed velocity $\boldsymbol{v}_{x}^{\prime}-\boldsymbol{v}_{x}$ and the term $\hat{\boldsymbol{v}}_{x} \boldsymbol{\alpha}_{x}$ (the cross product of the orientation error $\boldsymbol{\alpha}_{x}$ and velocity vector $\left.\boldsymbol{v}_{x}\right)$, i.e.

$$
\delta \boldsymbol{v}_{X}=\boldsymbol{v}_{X}^{\prime}-\boldsymbol{v}_{X}+\hat{\boldsymbol{v}}_{X} \boldsymbol{\alpha}_{X} .
$$

Then the INS error propagation equations for the velocity error $\delta \boldsymbol{v}_{x}$ and orientation error $\boldsymbol{\alpha}_{x}$ can be written as

$$
\begin{gathered}
\delta \dot{\boldsymbol{v}}_{x}=\left(\hat{\boldsymbol{\omega}}_{x \xi}^{x}+\hat{\boldsymbol{\omega}}_{\eta \xi}^{x}\right) \delta \boldsymbol{v}_{x}+\hat{\gamma}_{x} \boldsymbol{\alpha}_{x}-\delta \boldsymbol{g}_{x}+\Delta \boldsymbol{f}_{x}-\hat{\boldsymbol{v}}_{x}^{\prime} \Delta \boldsymbol{\omega}_{z \xi}^{x}, \\
\dot{\boldsymbol{\alpha}}_{x}=\hat{\boldsymbol{\omega}}_{x \xi}^{x} \boldsymbol{\alpha}_{x}-\Delta \boldsymbol{\omega}_{z \xi}^{x}
\end{gathered}
$$

where $\hat{\gamma}_{x}, \hat{\boldsymbol{v}}_{x}^{\prime}$ are the skew-symmetric matrices formed by the components of $\gamma_{x}, v_{x}^{\prime}$, respectively, $\Delta \boldsymbol{f}_{x}, \Delta \boldsymbol{\omega}_{z \xi}^{x}$ are the sensor errors expressed in the frame $M x: \Delta \boldsymbol{f}_{x}=$ $L_{x z}^{\prime} \Delta \boldsymbol{f}_{z}, \Delta \boldsymbol{\omega}_{z \xi}^{x}=L_{x z}^{\prime} \Delta \boldsymbol{\omega}_{z \xi}^{z}$. Note the negative sign in front of $\delta g_{x}$. This is due to the same sign of the velocity vector $\boldsymbol{v}_{x}$ in Eq. (4). Note also the presence of the gyroscope error in Eq. (5), which is due to the presence of the term $\hat{\boldsymbol{v}}_{x} \boldsymbol{\alpha}_{x}$ in Eq. (4). Furthermore, note that the gravity gradient matrix does not show up in Eq. (5) since position errors are assumed to be zero assuming GNSS positioning solution of high accuracy. Note also that from the definition of the velocity error Eq. (4) it follows that the accelerometer measurements do not show up in Eq. (5).

Denote by $\boldsymbol{v}_{x}^{G N S S}$ the GNSS velocity of the aircraft relative to the Earth and by $\boldsymbol{q}_{x}^{v}=\boldsymbol{v}_{x}^{G N S S}-\boldsymbol{v}_{x}$ the GNSS velocity error. Let $\boldsymbol{y}_{x}$ be the velocity observation obtained as $\boldsymbol{y}_{x}=\boldsymbol{v}_{x}^{\prime}-\boldsymbol{v}_{x}^{G N S S}$. Then the observation model is written as

$$
\boldsymbol{y}_{X}=\delta \boldsymbol{v}_{X}-\hat{\boldsymbol{v}}_{X}^{\prime} \boldsymbol{\alpha}_{X}-\Delta \boldsymbol{q}_{X}^{v} .
$$

Note that $\boldsymbol{v}_{x}^{G N S S}$ instead of $\boldsymbol{v}_{x}^{\prime}$ can also be used in the product $\hat{\boldsymbol{v}}_{x}^{\prime} \boldsymbol{\alpha}_{x}$ in Eq. (7) in the case of high accuracy of the GNSS velocity solution.

Thus, the problem is to determine the GDV from Eqs. (5), (6) given positions of the gravimeter proof mass (from GNSS observations), the velocity observations Eq. (7), a model of the normal gravity $\gamma_{x}$, and measurements of the body-frame absolute angular velocity $\boldsymbol{\omega}_{z \xi}^{z^{\prime}}$ and the specific force $\boldsymbol{f}_{z}^{\prime}$.

As the gravity horizontal components $\delta g_{x_{1}}, \delta g_{x_{2}}$ are of special interest, we further consider only the equations for the horizontal components in Eqs. (5), (7). Hence, rewrite Eqs. (5), (6) as follows:

$$
\begin{aligned}
& \delta \dot{v}_{x_{1}}=-\gamma_{0} \alpha_{x_{2}}-\delta g_{x_{1}}+\Delta f_{x_{1}}+v_{x_{2}}^{\prime} \Delta \omega_{x \xi}^{x_{3}}, \\
& \delta \dot{v}_{x_{2}}=\gamma_{0} \alpha_{x_{1}}-\delta g_{x_{2}}+\Delta f_{x_{2}}-v_{x_{1}}^{\prime} \Delta \omega_{x \xi}^{x_{3}}, \\
& \dot{\alpha}_{x_{1}}=\omega_{x \xi}^{x_{3}} \alpha_{x_{2}}-\omega_{x \xi}^{x_{2}} \alpha_{x_{3}}-\Delta \omega_{x \xi}^{x_{1}}, \\
& \dot{\alpha}_{x_{2}}=\omega_{x \xi}^{x_{1}} \alpha_{x_{3}}-\omega_{x \xi}^{x_{3}} \alpha_{x_{1}}-\Delta \omega_{x \xi}^{x_{2}}, \\
& \dot{\alpha}_{x_{3}}=\omega_{x \xi}^{x_{2}} \alpha_{x_{1}}-\omega_{x \xi}^{x_{1}} \alpha_{x_{2}}-\Delta \omega_{x \xi}^{x_{3}},
\end{aligned}
$$


where the small terms $\left(\hat{\boldsymbol{\omega}}_{x \xi}^{x}+\hat{\boldsymbol{\omega}}_{\eta \xi}^{x}\right) \delta \boldsymbol{v}_{x}, v_{x_{3}}^{\prime} \Delta \omega_{x \xi}^{x_{1}}, v_{x_{3}}^{\prime} \Delta \omega_{x \xi}^{x_{2}}$ are neglected (as highly accurate INS is assumed). Here $\gamma_{0}$ denotes the magnitude of the normal gravity vector $\gamma_{x}$. The corresponding observation equations are written as

$$
\begin{aligned}
& y_{x_{1}}=\delta v_{x_{1}}+v_{x_{2}}^{\prime} \alpha_{x_{3}}-q_{x_{1}}^{v}, \\
& y_{x_{2}}=\delta v_{x_{2}}-v_{x_{1}}^{\prime} \alpha_{x_{3}}-q_{x_{2}}^{v}
\end{aligned}
$$

where the small terms $v_{x_{3}}^{\prime} \alpha_{x_{1}}$ and $v_{x_{3}}^{\prime} \alpha_{x_{2}}$ are also neglected. The GNSS velocity errors $q_{x_{1}}^{v}, q_{x_{2}}^{v}$ are assumed to be zero-mean white-noise processes with known variances.

We consider simplified models for the gyroscope and accelerometer errors (for more detailed models see, e.g. Kwon and Jekeli (2001); Farrell (2008)):

$$
\Delta \boldsymbol{\omega}_{z \xi}^{z}=\boldsymbol{b}^{g}+\boldsymbol{q}^{g}, \Delta \boldsymbol{f}_{z}=\boldsymbol{b}^{a}+\boldsymbol{q}^{a}
$$

where $\boldsymbol{b}^{g}, \boldsymbol{b}^{a}$ are $3 \times 1$-vectors of gyroscope and accelerometer biases, respectively, $\boldsymbol{q}^{g}, \boldsymbol{q}^{a}$ are random errors. The biases are modeled as follows:

$$
\dot{\boldsymbol{b}}^{g}=\boldsymbol{q}^{b}, \dot{\boldsymbol{b}}^{a}=0 .
$$

The vectors $\boldsymbol{q}^{b}, \boldsymbol{q}^{g}, \boldsymbol{q}^{a}$ are assumed to be zero-mean white-noise processes with known variances. Note that different models are used for the gyroscope and accelerometer biases in Eq. (11). We assume that the accelerometer bias changes insignificantly over time (due to thermal control), e.g. about $0.2 \mathrm{mGal} /$ day as for the GT-1A gravimeter (Berzhitsky et al. 2002).

\section{The anomalous gravity field model as a random field}

To determine the GDV horizontal components from Eqs. (8)-(11), an additional information (or a hypothesis) on gravity should be introduced. Our approach is based on taking into account the spatial behavior of the anomalous gravity field. For this purpose, we use 2-D stochastic modeling of gravity. Preliminary theoretical analysis showed that gravity spatial correlations taken into account could significantly strengthen observability of the GDV horizontal components (Bolotin and Vyazmin 2018).

To simplify presentation, we make some assumptions regarding survey lines. Consider a set of lines of a typical airborne survey. Assume that the lines are equally spaced and flown at a constant height $h$ above the reference ellipsoid. Assume also that the lines are flown in the same direction (for example, from south to north) and the aircraft speed is constant at the lines. Consider a subset of $K$ neighbouring lines $\Gamma_{1}, \ldots, \Gamma_{K}$ of equal lengths (we will call this subset a block).

Let $x_{1}, x_{2}$ be orthogonal coordinates on the plane, which is fitted to the set of lines. Let the $x_{1}$-axis be directed across the lines (from west to east) and the $x_{2}$-axis be directed along the lines (from south to north). Denote by $\Delta x_{1}$ the line spacing and by $k$ the line number. Airborne measurements along the lines are assumed to be time-synchronized. Let $\Delta x_{2}$ be the spatial resolution of airborne measurements along a line, $i$ be the number of a measurement point on a line, $i=1, \ldots, I$, where $I$ is the total number of measurement points on a line. Thus there is a rectangular grid of the measurement points $(k, i) \sim$ $\left(k \Delta x_{1}, i \Delta x_{2}\right)$ at the flight height (see Fig. 1).

Denote by $T(k, i)$ values of the disturbing potential $T\left(k \Delta x_{1}, i \Delta x_{2}, h\right)$ in the knots $(k, i)$ of the grid. We use a stochastic model of the disturbing potential assuming that $T\left(k \Delta x_{1}, i \Delta x_{2}, h\right)$ is a homogeneous discrete twodimensional random field with the 2-D PSD expressed as the product of two 1-D PSD in the following form:

$$
\begin{aligned}
& S_{T}\left(u_{1}, u_{2}, h\right)=\frac{\sigma^{2}}{4 \pi^{2}}\left|H_{1}\left(e^{j u_{1} \Delta x_{1}}\right)\right|^{2}\left|H_{2}\left(e^{j u_{2} \Delta x_{2}}\right)\right|^{2} \\
& H_{1}\left(z_{1}\right)=\frac{1}{1-a_{1} z_{1}^{-1}-a_{2} z_{1}^{-2}}, H_{2}\left(z_{2}\right)=\frac{1}{1-c_{1} z_{2}^{-1}-c_{2} z_{2}^{-2}}
\end{aligned}
$$

where $a_{1}, a_{2}, c_{1}, c_{2}$ are the coefficients of the model, $\sigma^{2}$ is the variance of the random field, $u_{1}, u_{2}$ are spatial frequencies corresponding to $x_{1}, x_{2}$, respectively. Here $z_{1}, z_{2}$ are complex numbers, $j=\sqrt{ }-1$.

It follows from Eq. (12) that the disturbing potential can be represented by two 2nd-order autoregressive equations, one of which is defined on the $x_{1}$-axis and the other on the $x_{2}$-axis :

$$
\begin{gathered}
T(k, i)=c_{1} T(k, i-1)+c_{2} T(k, i-2)+w(k, i), \\
w(k, i)=a_{1} w(k-1, i)+a_{2} w(k-2, i)+q^{w}(k, i) .
\end{gathered}
$$

Here $q^{w}(k, i)$ is is the discrete-time white noise with zero mean and the variance $\sigma^{2}$. The coefficients $a_{1}, a_{2}, c_{1}, c_{2}$ can be computed given the a priori correlation radius of the anomalous gravity field. The along-line PSD and autocorrelation function of the gravity model are shown in Fig. 2. Figure 3 shows contours of the 2-D PSD of the gravity model (the model is nearly isotropic).

The gravity horizontal components can be expressed as

$$
\begin{aligned}
& \delta g_{x_{1}}(k, i) \approx \frac{1}{\Delta x_{1}}(T(k, i)-T(k-1, i)), \\
& \delta g_{x_{2}}(k, i) \approx \frac{1}{\Delta x_{2}}(T(k, i)-T(k, i-1)) .
\end{aligned}
$$




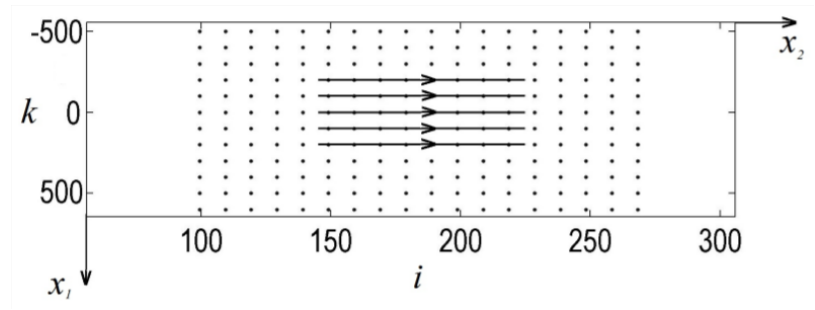

Fig. 1. Grid of knots and fragments of survey lines, $m \times m$.
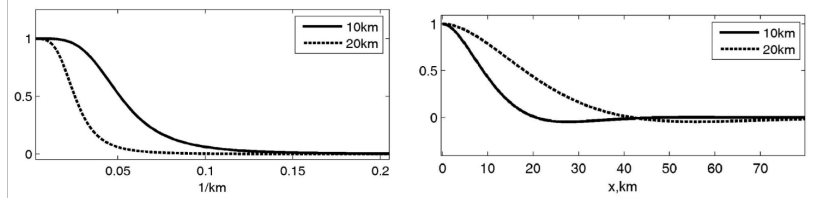

Fig. 2. The normalized along-line PSD (left) and autocorrelation function (right) for the correlation length $10 \mathrm{~km}$ (solid line) and $20 \mathrm{~km}$ (dashed line).

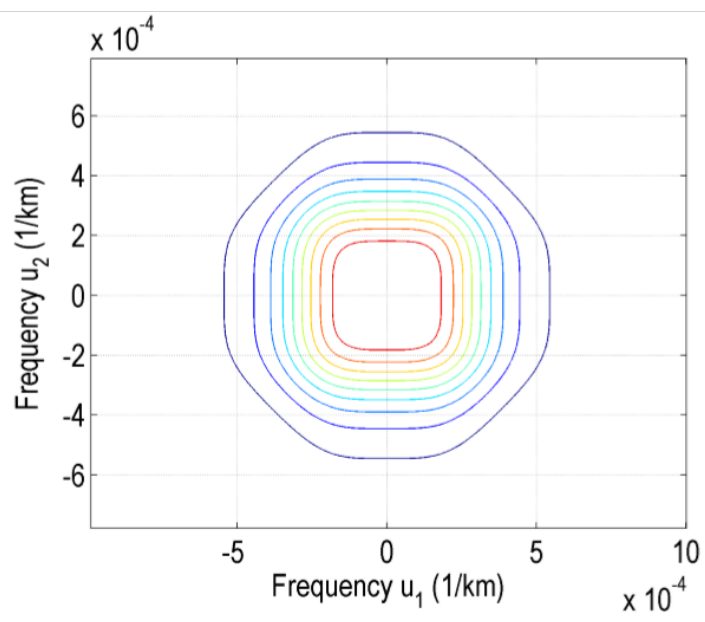

Fig. 3. Contours of the 2-D PSD of the stochastic model of the anomalous gravity field.

\section{Algorithm of the gravity horizontal component estimation}

Let us select a block of lines, i.e., a subset of $K$ neighbouring lines $\Gamma_{1}, \ldots, \Gamma_{K}$ of equal length. Consider the set of $K$ problems, each of which is described by Eqs. (8)-(9) and posed for one of $K$ neighbouring lines $\Gamma_{k}$. Introduce the $K \times 1$ - vector of values of a variable at the same measurement point $x_{2}=i \Delta x_{2}$ of each neighbouring line as follows (for example, for $\delta v_{1}$ ):

$$
\delta \boldsymbol{v}_{1}(i)=\left(\delta v_{1}\left(\Delta x_{1}, i \Delta x_{2}\right), \ldots, \delta v_{1}\left(K \Delta x_{1}, i \Delta x_{2}\right)\right)^{T}
$$

where $\delta v_{1}\left(k \Delta x_{1}, i \Delta x_{2}\right)$ denotes the value of the velocity error at the measurement point $x_{2}=i \Delta x_{2}$ at the line $\Gamma_{k}$.
For simplicity of notation, we omit the subscript $x$ hereinafter. Then we combine $K$ systems of the INS error equations Eq. (8) and $K$ systems of the observation equations Eq. (9) into one system that can be written briefly in the discrete-time form as

$$
\begin{aligned}
& \boldsymbol{x}(i+1)=A(i) \boldsymbol{x}(i)+B(i) \boldsymbol{q}(i), \\
& \boldsymbol{y}(i)=C(i) \boldsymbol{x}(i)+\boldsymbol{r}(i),
\end{aligned}
$$

where the state vector $x(i)$ includes the following 13 variables (each one is a $K \times 1$-vector) at the $i$-th time instant (the total dimension is $13 \mathrm{~K} \times 1$ ):

$$
\begin{aligned}
& \delta \boldsymbol{v}_{1}(i), \delta \boldsymbol{v}_{2}(i), \boldsymbol{\alpha}_{1}(i), \boldsymbol{\alpha}_{2}(i), \boldsymbol{\alpha}_{3}(i), \boldsymbol{T}(i), \boldsymbol{T}(i-1), \\
& \boldsymbol{b}_{1}^{a}(i), \boldsymbol{b}_{2}^{a}(i), \boldsymbol{b}_{3}^{a}(i), \boldsymbol{b}_{1}^{g}(i), \boldsymbol{b}_{2}^{g}(i), \boldsymbol{b}_{3}^{g}(i) .
\end{aligned}
$$

The matrices $A(i), B(i), C(i)$ in Eq. (16) are formed by the coefficients of the INS error equations and the observation equations, $\boldsymbol{q}(i)$ is a random vector formed by the inertial sensor noises $\boldsymbol{q}^{a}, \boldsymbol{q}^{g}, \boldsymbol{q}^{b}$ and the noise $q^{w}$ from the gravity model Eq. (14), $\boldsymbol{r}(i)$ is the random vector formed by the GNSS velocity error $\boldsymbol{q}^{v}$. We assume that $\boldsymbol{q}(i), \boldsymbol{r}(i)$ are white noise processes with zero mean and known covariance matrices.

We now can pose the standard optimal estimation problem (under the minimum mean squared error criterion) for the system's state vector Eq. (17) given the state space model Eq. (16) and the covariance matrices for the random vectors $\boldsymbol{q}(i), \boldsymbol{r}(i)$.

The estimation algorithm is based on Kalman filtering and includes the following steps. At the first step, the posed estimation problem for the block of lines (i.e., the set of $K$ neighbouring lines of equal length) is solved via Kalman filtering. At the second step, the Rauch-TungStriebel (RTS) smoother (Kailath et al. 2000) is applied to the Kalman filter estimates. The GDV horizontal component estimates are obtained from the disturbing potential estimate. At the next step, the following block of lines (i.e., the set of lines $\left.\Gamma_{2}, \ldots, \Gamma_{K+1}\right)$ is selected and the steps 1 and 2 are repeated.

In the case when $K$ is equal to the total number of survey lines, the estimation algorithm is optimal in the mean squared error sense in the class of linear algorithms. The number of the adjacent lines should be greater or equal to 3. Data flow diagram of the developed algorithm is shown in Fig. 4. 


\section{Data processing results and analysis}

\section{A. Data simulation}

The developed algorithm was applied to simulated airborne gravimetry data. The inertial navigation simulator (Bogdanov and Golovan 2017) was used that provided a flight path and readings of a virtual inertial measurement unit (IMU). Flight path parameters (coordinates, velocity, attitude angles) and error-free IMU measurements were simulated along 8 parallel survey lines at a constant height of $570 \mathrm{~m}$ above the reference ellipsoid. The lines are directed from south to north and spaced at $800 \mathrm{~m}$. Initial latitude and longitude for the line 1 are $43^{\circ}$ and $32^{\circ}$, respectively. Length of each line equals $90 \mathrm{~km}$. An aircraft flying speed along a line was $100 \mathrm{~m} / \mathrm{s}$. The yaw, roll and pitch angles were constant along each line (the roll and pitch were set to 0$)$.

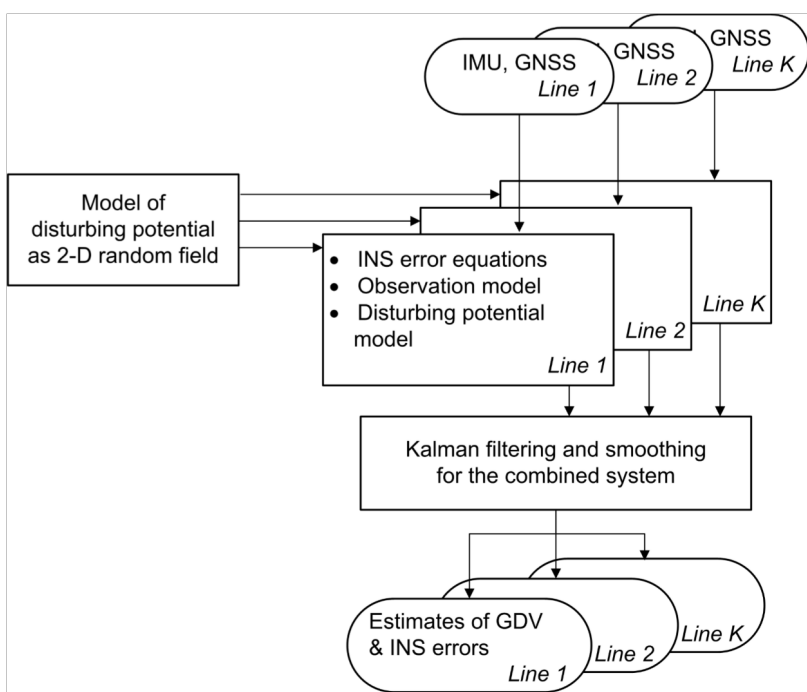

Fig. 4. Data flow diagram for the GDV estimation along adjacent lines.

The anomalous gravity field at the flight height was simulated as a random field generated by two autoregressive equations of order 3 (in a manner similar to generating the model of order 2 given by Eqs. (13)-(14)). Then the generated random field was smoothed by the 2-D Hann window to reduce anisotropic effects. Note that the autoregressive equations of a different order were used at the data simulation stage than those (Eqs. (13)-(14)) used in the estimation algorithm. A different order of the autoregressive equations was used as otherwise accuracy of the final estimates of the GDV could be unreasonably optimistic.

The gravity correlation radius is about $5 \mathrm{~km}$. Figure 5 shows the along-line GDV components derived from the simulated anomalous gravity field. Figure 6 shows maps of the simulated gravity components in the survey area.

To simulate gyroscope measurement errors (noise and bias drift), we used readings of a calibrated gyroscope triad (fiber optic gyroscopes (FOG) by Optolink, LLC), which were recorded during a standstill test. The Allan variances calculated from the gyroscope readings are shown in Fig. 7. Bias instability was around $0.002-0.004^{\circ} / \mathrm{h}$. Standard deviation of noise (angular random walk) equaled $0.1^{\circ} / \mathrm{h} / \sqrt{ } \mathrm{Hz}$. The error-free gyro readings provided by the simulator were perturbed by an additive zero-mean noise process derived from the FOG readings and by a constant bias of $0.005^{\circ} / \mathrm{h}$.
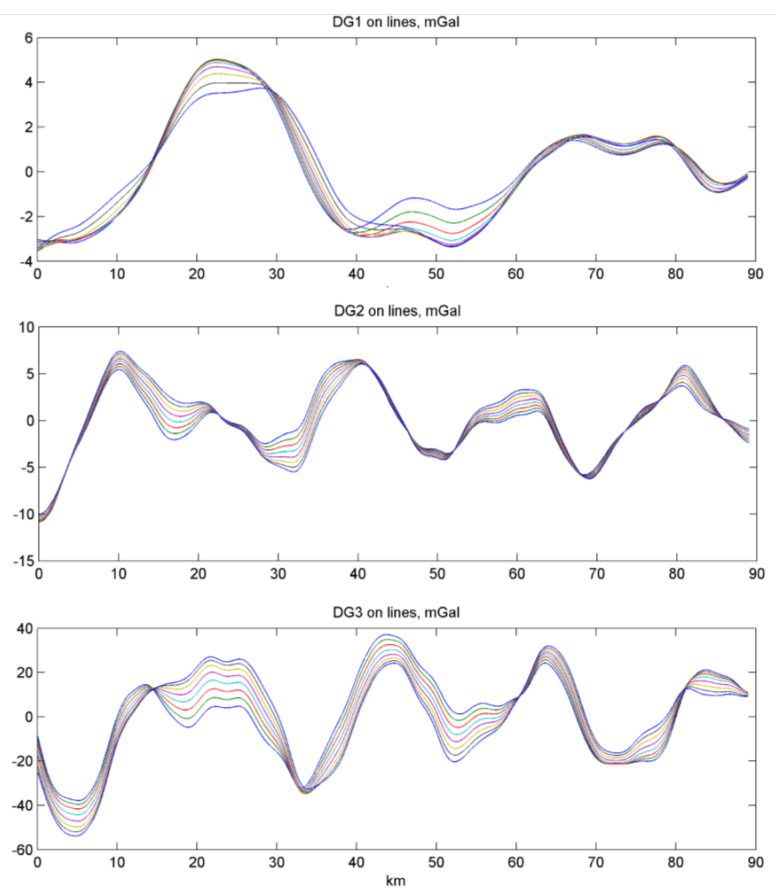

Fig. 5. Gravity disturbance components (simulated data) along 8 survey lines: east (top), north (middle), up (bottom), [mGal].

To simulate accelerometer errors, an additive zeromean white-noise process with standard deviation of $1.5 \mathrm{mGal} / \sqrt{ } \mathrm{Hz}$ was simulated. Then the constant bias of 10 mGal was added to accelerometer measurements. No bias drift was simulated assuming the presence of a highly stable thermal control as, e.g., in Berzhitsky et al. (2002).

The GNSS velocity solution was assumed to have only an additive random-noise error, which was simulated as the first-order difference of a zero-mean white-noise pro- 

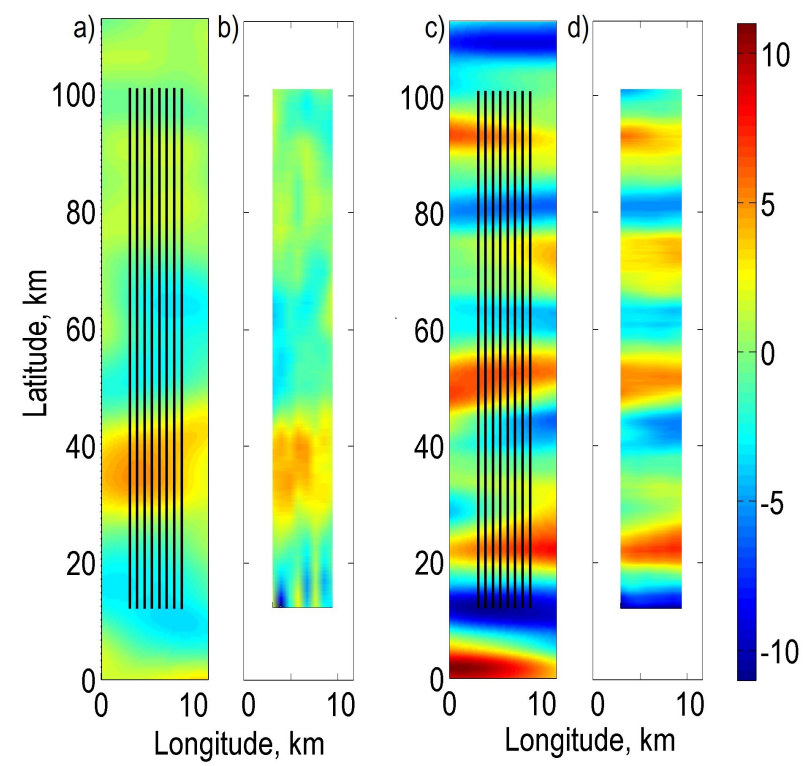

Fig. 6. True gravity components (simulated data) and the estimates provided by the algorithm based on 2-D autoregressive gravity model: a) true (east), b) the estimate (east), c) true (north), d) the estimate (north), [mGal].

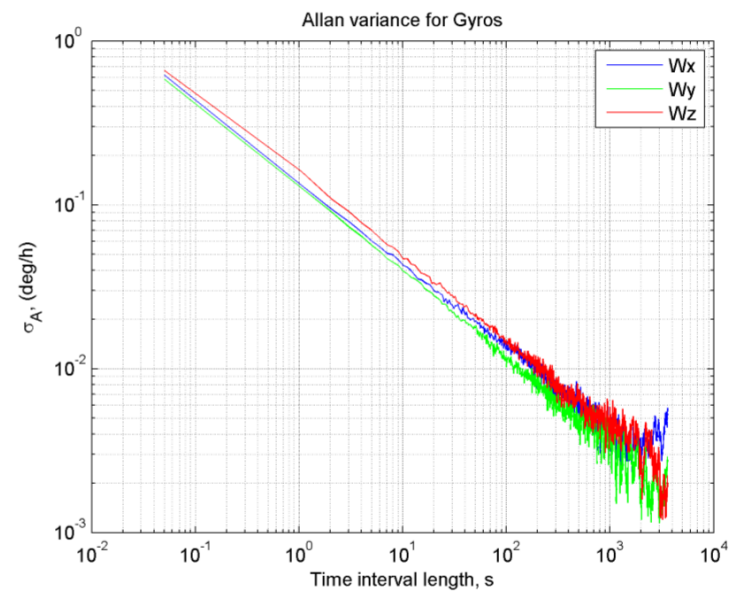

Fig. 7. Square root of the Allan variances calculated from the readings of the gyroscope triad (FOG by Optolink), [ $\% / h]$.

cess at $20 \mathrm{~Hz}$. The standard deviation of the simulated GNSS velocity error equaled $3 \mathrm{~mm} / \mathrm{s} / \sqrt{ } \mathrm{Hz}$ [the value can be found, e.g., in Freda et al. (2015)].

\section{B. Data processing}

The inertial sensor measurements and GNSS data simulated along 8 survey lines were processed by the proposed algorithm. First, the INS mechanization equations Eq. (3) were solved for all points along the survey lines given known initial values for $\boldsymbol{v}_{x}^{\prime}$ and $L_{z x}$ for each line (precise initial alignment was assumed).

After that, the disturbing potential and INS errors were estimated along all 8 lines simultaneously via the Kalman filter (open-loop estimation). The coefficients of the autoregressive equations Eqs. (13)-(14) were preliminarily computed for the a priori value of the gravity correlation radius equaled to $5 \mathrm{~km}$. The state vector Eq. (17) of the combined system Eq. (16) included the velocity errors, the attitude errors, the inertial sensor biases, and the disturbing potential values at the $i$-th measurement point of each line. The total number of the variables of the state vector equaled 104.

At the next step, the estimates provided by the Kalman filter were smoothed by applying the RTS-smoother. Then the along-line estimates of the GDV horizontal components were obtained using the formula Eq. (15).

\section{Results}

Results of the gravity horizontal component estimation are shown in Fig. 6. Errors of the GDV estimation obtained as the difference of the along-line estimates and the true data are shown in Fig. 8. The accuracy of the gravity horizontal component estimation $(1 \sigma)$ ranges from 0.9 to $2.1 \mathrm{mGal}$ for the east component and from 0.7 to $1.2 \mathrm{mGal}$ for the north component. Standard deviation of the estimate error over all 8 lines equals $1.43 \mathrm{mGal}$ for the east component and $0.97 \mathrm{mGal}$ for the north component.

Loss of accuracy of the gravity estimates can be observed at the edges of the processed area (Fig. 8). This effect was expected as it followed from the properties of the gravity autoregressive model used by the algorithm. The estimate error is less than $4 \mathrm{mGal}$ inside the area and less than $8 \mathrm{mGal}$ for the whole area (for both components).

The constant biases in outputs of the horizontal gyroscopes were estimated with good accuracy: mean value of the along-line estimates ranges from 0.003 to $0.006^{\circ} / \mathrm{h}$ (the true value is $0.005^{\circ} / \mathrm{h}$ ). The estimates of the constant biases of the vertical gyroscope and the accelerometers are inaccurate as these are almost unobservable. The horizontal orientation errors $\alpha_{x_{1}}, \alpha_{x_{2}}$ are less than 6 " for each survey line. These errors were estimated with the accuracy of about $0.4^{\prime \prime}(1 \sigma)$ and $0.4^{\prime \prime}-2.2^{\prime \prime}$ (mean value).

Table 1 summarizes statistics of the along-line gravity estimate errors. Mean value of the estimate error for the north component is small and reaches only $0.04 \mathrm{mGal}$. However, mean value of the east component estimate error is larger and reaches $0.2-0.4 \mathrm{mGal}$ for most lines. The 
Table 1. Numerical results with the proposed algorithm based on the 2-D autoregressive gravity model: mean value and standard deviation of the gravity estimate errors, $\mathrm{mGal}$.

\begin{tabular}{llllllllll}
\hline Gravity component & Line 1 & Line 2 & Line 3 & Line 4 & Line 5 & Line 6 & Line 7 & Line 8 & All lines \\
\hline East (SD) & 1.54 & 1.38 & 0.86 & 1.68 & 0.89 & 1.65 & 1.39 & 2.06 & 1.43 \\
North (SD) & 1.11 & 1.15 & 1.11 & 1.02 & 0.97 & 0.90 & 0.79 & 0.69 & 0.97 \\
East (mean) & -0.05 & -0.37 & -0.03 & 0.27 & -0.24 & 0.21 & -0.28 & -0.01 & -0.06 \\
North (mean) & 0.01 & 0.10 & 0.09 & 0.03 & 0.02 & -0.03 & -0.03 & -0.04 & 0.02 \\
\hline
\end{tabular}

reason is likely that some unobservable long-wavelength parts remained in the estimate of the east component.

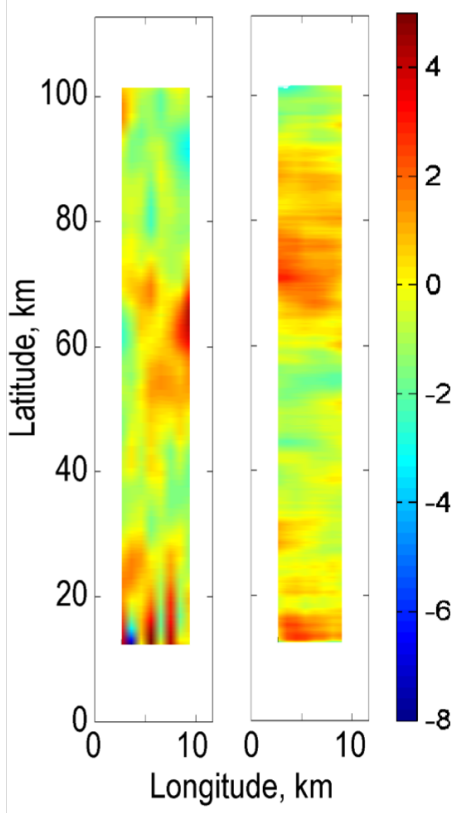

Fig. 8. Differences of the estimated GDV components and true data: east component (left) and north component (right), [mGal].

\section{1-D autoregressive gravity model}

For the purposes of comparison, the GDV horizontal components were also estimated using the traditional approach based on time-domain stochastic modeling of the GDV components. We used a third-order Gauss-Markov model for the gravity components, which is written in the time domain as

$$
\delta g_{m}^{(3)}+3 \beta_{g} \delta \ddot{g}_{m}+3 \beta_{g}^{2} \delta \dot{g}_{m}+\beta_{g}^{3} \delta g_{m}=q_{m}^{\mathrm{g}},
$$

where $m=1,2, q_{m}^{\mathrm{g}}$ is a zero-mean white noise with variance $\sigma_{q}^{2}=\frac{16}{3} \beta_{g}^{5} \sigma_{g}^{2}$. The following values for the gravity model parameters were adopted: $50 \mathrm{~s}$ for the correlation time $1 / \beta_{g}$ (equivalent to $5 \mathrm{~km}$ ) and $5 \mathrm{mGal}$ for $\sigma_{g}$.
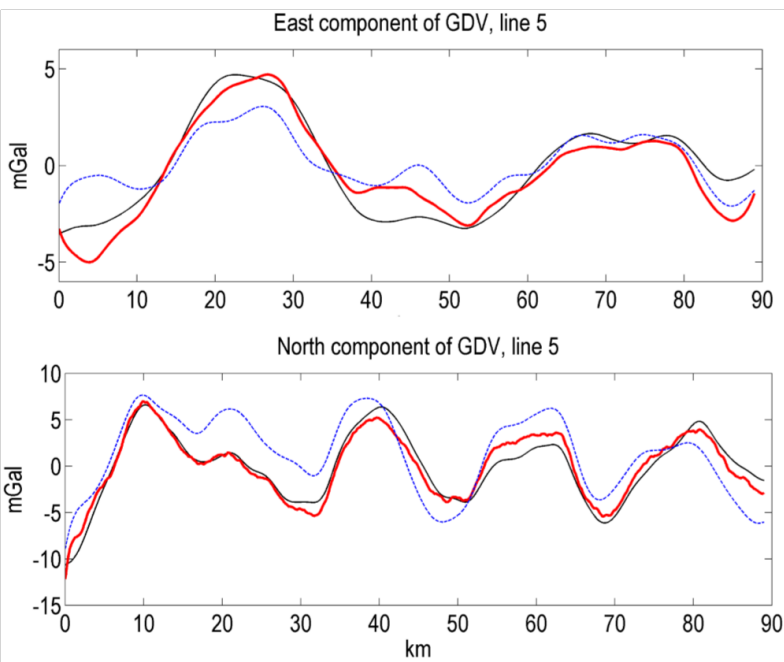

Fig. 9. East and north components of the gravity disturbance along the line 5: true (black), the estimate provided by the proposed algorithm based on the 2- D autoregressive gravity model (red), the estimate provided by the standard algorithm based on the 1-D autoregressive gravity model (blue), [mGal].

Hence, the gravity horizontal components and INS systematic errors can be estimated along a line given the state space model written in the continuous-time form Eqs. (8)-(11) and (18). The system's state vector includes 17 variables, which are the INS errors:

$$
\delta v_{1}, \delta v_{2}, \alpha_{1}, \alpha_{2}, \alpha_{3}, b_{1}^{a}, b_{2}^{a}, b_{3}^{a}, b_{1}^{g}, b_{2}^{g}, b_{3}^{g},
$$

and the gravity model parameters:

$$
\delta g_{1}, \delta \dot{g}_{1}, \delta \ddot{g}_{1}, \delta g_{2}, \delta \dot{g}_{2}, \delta \ddot{g}_{2} .
$$

The along-line gravity estimates were obtained via Kalman filtering (the open-loop estimation) and then smoothed using the RTS-smoother.

Only two lines (No. 4 and No. 5) were selected for processing and comparing with the new approach. The reason is that the new approach is expected to provide the most accurate estimates for the lines in the centre of the block of the lines. The other lines in the block are auxiliary for estimating gravity along the central lines. It should be noted that the new approach is intended for processing datasets with a large number of lines. The lines of a such dataset are 
assumed to be represented as a set of overlapping blocks of lines, where each block consists of $K$ neighbouring lines of equal length. At the first step, the first block is processed, then the second one, and so forth (see Sect. 4). At each step, the result for the line in the centre of a block is stored. The number $K$ of the lines in a block is selected by the user and is assumed to be much smaller than the total number of lines.

The gravity component estimates along line 5 obtained using the two approaches are shown in Fig. 9. The traditional approach showed less accurate estimates of the horizontal components than the approach based on the 2-D autoregressive gravity model (see Fig. 9). Non-linear trends caused by the orientation errors and the sensor biases can be observed in the estimates. The accuracy of the estimates provided by the traditional approach is $30 \%$ (line 4) and 50\% (line 5) worse for the east component and $66 \%$ (line 4) and almost 300\% (line 5) worse for the north component (see Table 2).

Mean value of the east component estimate error for line 5 is at the same level of $0.2 \mathrm{mGal}$ as in the case of using the new approach. However, mean value of the east component estimate error for line $4(0.11 \mathrm{mGal})$ is 2.5 times better than in the case of using the new approach $(0.27 \mathrm{mGal})$. Mean value of the north component estimate error equals $0.64 \mathrm{mGal}$ (line 4) and $1.36 \mathrm{mGal}$ (line 5) when using the traditional approach. Both values are significantly worse than those obtained using the new approach $(0.03 \mathrm{mGal}$ for line 4 and $0.02 \mathrm{mGal}$ for line 5).

Table 2 summarizes statistics for the errors of the gravity estimates along line 4 and line 5 obtained using the traditional approach.

Table 2. Numerical results with the algorithm based on 1-D autoregressive gravity model (the third-order Gauss-Markov process): mean value and standard deviation of the gravity estimate errors, mGal.

\begin{tabular}{lll}
\hline Gravity component & Line 4 & Line 5 \\
\hline East (SD) & 2.18 & 1.42 \\
North (SD) & 1.69 & 2.78 \\
East (mean) & 0.11 & 0.23 \\
North (mean) & 0.64 & 1.36 \\
\hline
\end{tabular}

\section{Conclusions}

An approach to determination of the gravity disturbance horizontal components from airborne measurements at a set of parallel survey lines is proposed. The approach is based on using a 2-D homogeneous random field for the gravity field modeling. The random field is generated by a combination of two autoregressive equations of order two, one of which is defined along a survey line and the other across the lines. The developed estimation algorithm is based on Kalman filtering and smoothing techniques. Several adjacent parallel survey lines are selected for simultaneous processing. The developed estimation algorithm is optimal under the minimum mean squared error criterion in the class of linear algorithms as the number of selected adjacent lines tends to the total number of survey lines.

Simulated airborne data were processed by the developed algorithm. A navigation-grade IMU and the differential mode of GNSS were assumed.

The new approach showed significant reduction of the impact of the IMU sensor and orientation errors on the gravity horizontal component estimation. Numerical results showed the estimation accuracy of $1.43 \mathrm{mGal}$ and 0.97 $\mathrm{mGal}$ ( $1 \sigma$ over all processed lines) for the east and north components, respectively. However, the results may be optimistic for airborne vector gravimetry as the simplified models were used for simulating observation errors.

The new approach was compared with the traditional one based on representing each gravity component by a stochastic process in the time domain. The accuracy of the best estimate obtained using the new approach (along the line in the centre of all lines) is $0.89 \mathrm{mGal}$ for the east and $0.97 \mathrm{mGal}$ for the north component $(1 \sigma)$. This is several times better than the accuracy achieved using the traditional approach.

The bias in the north component estimates computed using the new approach is small (less than $0.04 \mathrm{mGal}$ ) in comparison with the bias in the east component (at the level of $0.2-0.4 \mathrm{mGal}$ for most lines). An external information (such as lines in cross-track direction or a global gravity field model) is likely to be required when using the new approach, and this is the subject for further investigation.

The proposed approach has general requirements for survey lines (such as equal spacing, constant height above the reference ellipsoid, and constant cruising speed) and does not require repeated lines. There is also a less general requirement such as the same flight direction for each line. However, this can be avoided by solving the INS mechanization equations backward in time for the lines flown in opposite direction.

In conclusion, it should be also noted that the sensitivity of the proposed approach to the order of the random field model, the a priori gravity correlation ra- 
dius and the variance of the generating noise is of interest.

Acknowledgements: The authors would like to thank the reviewers for their very valuable comments.

This work was supported by the Russian Foundation for Basic Research (grant number 16-01-00731).

\section{References}

Becker D., Becker M., Olesen A.V., Nielsen J.E. and Forsberg R., 2016, Latest results in strapdown airborne gravimetry using an iMAR RQH unit, In: Proc. of the 4th IAG Symposium on Terrestrial Gravimetry: Static and Mobile Measurements, Consern CSRI Elektropribor, St. Petersburg, 19-25.

Berzhitsky V.N., Bolotin Yu.V., Golovan A.A., Ilyin V.N., Parusnikov N.A., Smoller Y.L. and Yurist S.S, 2002, GT-1A inertial gravimeter system. Results of flight tests, Lomonosov Moscow State University, Faculty of Mechanics and Mathematics, Moscow, Russia.

Bolotin Yu. V. and Golovan A. A., 2013, Methods of inertial gravimetry, Moscow Univ. Mech. Bull., 68, 5, 117-125.

Bolotin Yu. V. and Vyazmin V. S, 2018, Accuracy analysis of the airborne vector gravimetry algorithm based on two-dimensional random fields for gravity modeling, In: Proc. of the 25 th Anniversary Saint Petersburg International Conference on Integrated Navigation Systems. Consern CSRI Elektropribor, St. Petersburg, 179 - 183.

Cai S., Zhang K. and Wu M., 2013, Improving airborne strapdown vector gravimetry using stabilized horizontal components, J. Appl. Geoph., 98, 79-89.

Childers V. A., Bell R. E. and Brozena J. M., 1999, Airborne gravimetry: An investigation of filtering, Geoph., 64, 1, 61-69.

Dai D., Wang X., Zhan D. and Huang Z., 2014, An improved method for dynamic measurement of deflections of the vertical based on the maintenance of attitude reference. Sensors, 14, 1632216342.

Forsberg R., 1987, A new covariance model for inertial gravimetry and gradiometry, J. Geoph. Res., 92, B2, 1305-1310.

Bogdanov O. N. and Golovan A. A., 2017, Application of GNSS-INS simulator for testing algorithms of the airborne vector gravimetry problem, In: Proc. of the 11 th International Conference on Mathematical Problems in Engineering, Aerospace and Sciences, 1798.

Farrell J. A., 2008, Aided navigation systems: GPS and high rate sensors, New York, NY, McGraw-Hill.

Freda P., Angrisano A., Gaglione S. and Troisi S., 2015, Timedifferenced carrier phases technique for precise GNSS velocity estimation, GPS Solut, 19, 2, 335-341.

Huddle J. R., 1983, Inertial navigation system error-model considerations in Kalman filtering applications, Control Dyn. Syst., 20, 293-338.

Jekeli C., 1994, Airborne vector gravimetry using precise, positionaided inertial measurement units, Bull. Geod., 69, 1, 1-11.

Jordan S. K., 1972, Self-consistent statistical models for the gravity anomaly, vertical deflections, and undulation of the geoid, J. Geophys. Res., 77, 20, $3660-3670$.
Kailath T., Sayed A. H. and Hassibi B., 2000, Linear estimation. Prentice Hall, Englewood Cliffs.

Kennedy S.L., Bruton A.M. and Schwarz K.P., 2002, Improving DGPS accelerations for airborne gravimetry: GPS carrier phase accelerations revisited, In: Vistas for Geodesy in the New Millennium, IAG Symposia, Springer, Berlin, Heidelberg.

Kwon J. and Jekeli C., 2001, A new approach for airborne vector gravimetry using GPS/INS, J. Geod., 74, 10, 690-700.

Lu B., Barthelmes F., Petrovic S., Förste C., Flechtner F., Luo Z. et al., 2017 , Airborne gravimetry of GEOHALO mission: data processing and gravity field modeling, JGR: Solid Earth, 122.

Mangold U., 1997, Theory and performance prediction for continuous vector gravimetry based on a DGPS augmented rate bias inertial navigation system and a star tracker, Navigation, 44, 3, 329-345.

Olson D., 2010, GT-1A and GT-2A airborne gravimeters: improvements in design, operation, and processing from 2003 to 2010, In: Proc. of the ASEG-PESA Airborne Gravity 2010 Workshop, Sydney, Australia.

Pavlis N. K., Holmes S. A., Kenyon S. C., Factor J. K., 2012, The development and evaluation of the Earth Gravitational Model 2008 (EGM2008), J. Geophys. Res., 117, B04406.

Schwarz K. P., Kern M. and Nassar S. M., 2002, Estimating the gravity disturbance vector from airborne gravimetry, In: Vistas for Geodesy in the New Millennium, IAG Symposia, Springer, Berlin, Heidelberg. 